\title{
Repetitive Control of Electrical Stimulation for Tremor Suppression
}

\author{
Engin H. Copur, Chris T. Freeman, Bing Chu, and Dina S. Laila
}

\begin{abstract}
Tremor is a rapid involuntary movement often seen in patients with neurological conditions such as Multiple Sclerosis (MS) and Parkinson's disease. This debilitating oscillation can be suppressed by applying functional electrical stimulation (FES) within a closed-loop control system. However, conventional implementations use classical control methods and have proved capable of only limited performance. This paper establishes the feasibility of embedding repetitive control (RC) action to exploit the capability of learning from experience to completely suppress tremor at the wrist via FES regulated co-contraction of wrist extensors/flexors. A nonlinear model structure and associated identification procedure is first proposed to guarantee stability and performance of the RC system. Then a linearising control approach is developed to facilitate transparent $\mathrm{RC}$ design, together with a mechanism to preserve patients' voluntary intention. Experimental evaluation is performed with both unimpaired and neurologically impaired participants using a validated wristrig. For the former group a novel electromechanical system is employed to induce tremor artificially. Results are benchmarked against a well-known classical filtering technique to establish the efficacy of the RC approach. These confirm that the proposed control system with the developed model identification procedure can increase tremor suppression by $43.3 \%$ compared with conventional filtering. In addition, the mechanism decreases the interference of RC action with voluntary motion by $20.2 \%$ compared with conventional filtering.
\end{abstract}

Index Terms-Tremor suppression, functional electrical stimulation, repetitive control, induced tremor.

\section{INTRODUCTION}

$\mathbf{T}$ REMOR presents as a rhythmic, approximately periodic and involuntarily oscillation of a body part and manifests as a symptom of neurological disorders, occurring in over 50\% of Multiple Sclerosis (MS) and 75\% of Parkinson's disease cases. Intention tremor is frequently diagnosed within MS patients and occurs in the distal joints of the upper limb such as the wrist or fingers, and typically has a frequency between $2-5 \mathrm{~Hz}$ [1], [2]. Caused by malfunction of feedforward loops within the central nervous system [3], tremulous movement often results in serious impairment of functional abilities which may contribute to feelings of social isolation and depression [4]. Due to its prevalence and impact, suppression of tremor has been a popular research area for many years.

Invasive interventions to suppress tremor have inherent risks and are expensive [5]-[7], while medication has not provided effective treatment [8]. Other non-pharmacological

E. H. Copur, C. T. Freeman and B. Chu are with Electronics and Computer Science, University of Southampton, Southampton SO17 1BJ, U.K. (e-mail: \{ehc1g12,cf,b.chu\}@ecs.soton.ac.uk).

D. S. Laila is with the School of Engineering Sciences, University of Southampton, Southampton SO17 1BJ, U.K. (e-mail: D.Laila@soton.ac.uk).

Manuscript received Month Day, Year; revised Month Day, Year. methods include tremor suppressing orthoses [9], [10], limb cooling [11], vibration therapy [12] and adding limb weights [13]. However they also give rise to significant difficulties and adverse effects. For example, slowness and fatigue in performing the task are inevitable outcomes of limb cooling due to the slowing down of nerve conduction and muscle spindle activity. Moreover, such methods also cause a decrease in maximum achievable voluntary forces. Adding weights to relevant body parts also leads to accelerated muscle fatigue. Tremor suppressing orthoses are electromechanical systems comprised of actuators and sensors. The presence of these devices is extremely inconvenient when performing daily life activities, causing fatigue, obstructing range of movement, and generating feelings of self-consciousness due to their large size. Hence integration into patients' daily lives is challenging.

An alternative approach is to use functional electrical stimulation (FES) to suppress tremor. FES provides muscle contraction through artificial excitation of nerves. This contraction is triggered by low-level electrical impulses that are transmitted through surface electrodes adhered to the skin over the relevant muscles. This technique is generally applied in a closed-loop feedback arrangement where the electrical stimulation input stimulates muscles in anti-phase with respect to the tremulous motion. However effective suppression depends strongly on how accurately the level and timing of FES applied to the appropriate muscles are regulated by the controller.

In [14] conventional high-pass and band-pass filters were applied in closed-loop to suppress tremor at the wrist or elbow joint. Testing was conducted with MS patients who were divided into three groups according to their tremor type. Experimental results showed that essential, Parkinsonian and intention tremor could be reduced by $73 \%, 62 \%$ and $38 \%$, respectively. However, the model used to design the filters was not identified individually, resulting in poor control performance, particularly in intention tremor. In [15] a fuzzy logic controller combined with proportional-derivative (PD) action was developed. Simulation results showed that tremor was suppressed by $85 \%$ but were not supported by experimental evidence. In [16] a proportional-integral (PI) controller was implemented to alter joint impedance by means of FES to suppress tremor, and modest reduction was achieved. In [17] muscle co-contraction was used to increase the stiffness and viscosity of an antagonist muscle pair, thereby reducing tremor amplitude significantly. However, modifying muscle properties led to reducing the magnitude response of the joint at low frequencies so that more voluntary muscle force was needed to perform activities and this increasing demand resulted in greater muscle fatigue. All classical feedback systems have 
been found to modify the system's dynamic response below $1 \mathrm{~Hz}$, and thereby distort the intended motion to some extent. This effect is generally proportionate to the effort expended by the controller in suppressing the tremor, and may also be overshadowed by the perceived improvement in voluntary motion due to reduction in tremor at higher frequencies. Classical feedback systems also introduce large high-frequency stimulation transients that cause significant discomfort [18].

To enforce complete tremor suppression necessitates embedding an internal model of the periodic disturbance within the control structure prior to ensuring closed-loop asymptotic stability [19], which is equivalent to applying repetitive control (RC). This paper develops a rigorous, general framework for implementing RC based FES tremor suppression. This framework includes a linearising procedure, and a mechanism to preserve the patient's voluntary motion. This mechanism employs a general class of $\mathrm{RC}$, together with a zero-phase high-pass (ZPHP) filter to preserve voluntary movement at low frequencies and prevent FES from producing large highfrequency stimulation transients which would cause discomfort. Since RC requires a model of the underlying dynamics of the affected body part, this paper also provides a system identification procedure, prior to the derivation of appropriate $\mathrm{RC}$ forms [20]. To illustrate the framework and show its generality with respect to underlying $\mathrm{RC}$ forms, two $\mathrm{RC}$ algorithms are proposed and compared against classical feedback control design approaches. Experimental evaluation is undertaken using a wrist rig that has previously been validated to detect changes in upper limb function. In the first phase of this study tests are undertaken on neurologically intact participants, and therefore, an innovative electromechanical system is introduced to induce tremor artificially. Each participant completes three consecutive tests to quantify: (1) the effect of induced tremor on voluntary task completion, (2) the capability of FES to suppress induced tremor, and (3) the effect of FES on voluntary motion. In the second phase of the study, tests are undertaken on a neurologically impaired participant.

Note that a specific RC scheme was proposed for FESbased tremor suppression in [21]. This established feasibility, but substantial limitations in the model structure, identification procedure, and $\mathrm{RC}$ form meant that it is unsuitable for clinical deployment. The current paper rigorously addresses all these issues, and in particular embeds the novel contributions: (i) fully parameterised muscle and rigid body dynamics, (ii) multiple methods to incorporate voluntary motion, (iii) a validated identification procedure suitable for clinical use, (iv) a design procedure encompassing a broad class of RC schemes, (v) stability and robustness proofs with and without ZPHP filtering, (vi) comparative experimental results between multiple RC forms and the leading conventional design, (vii) evaluation on neurologically intact participants using a novel, improved method for artificially inducing tremor, and (viii) validation on a neurologically impaired participant to demonstrate effectiveness and potential for clinical deployment.

The paper is organized as follows: Section II presents a model and identification procedure to capture the relevant limb dynamics. Section III introduces a general RC design framework, and illustrates its application with two popular
RC approaches. Section IV further develops the framework to ensure the patient's voluntary intention is preserved. Section V summarises an experimental test procedure with results given in Section VI to evaluate the controllers. Section VII presents conclusions and directions for future research.

\section{TRemulous Wrist Model}

Previous FES tremor suppression research has almost exclusively focused on the wrist due to its importance in daily life activities and since intention tremor affects distal joints more strongly than proximal joints [22], [23]. The underlying dynamics of this joint in response to both tremor and FES are illustrated in Fig. 1 where FCR and ECR denote Flexor Carpi Radialis and Extensor Carpi Radialis muscles respectively. The model comprises rigid body dynamics characterising mass, inertia, damping and stiffness properties of limb segments, in combination with a Hill-type model representing the response of muscle to tremor and applied FES. Among a variety of models for the latter [24]-[27], a Hammerstein structure will be assumed due to its popularity in the literature [27], confirmed accuracy, structural simplicity, and correspondence with biophysics. The rigid body dynamics are commonly considered to exhibit linear elastic and damping, an assumption that has been experimentally verified in previous research [28]-[30]. This yields the following model definition:

Definition 1 (Wrist Model): Let the FCR muscle stimulated by input $u_{f c r}$ be modelled by a Hammerstein structure comprising a static mapping $h_{I R C, f c r}\left(u_{f c r}\right)$ followed by a linear activation dynamics $H_{L A D}(q)$. Let tremor present as a $N_{p^{-}}$ periodic signal $d_{f c r}$ which adds to the intermediate signal such that the overall moment $\tau_{f c r}=H_{L A D}(q)\left(h_{I R C, f c r}\left(u_{f c r}\right)+\right.$ $\left.d_{f c r}\right)$ produced by FCR muscles. Similarly, the overall moment $\tau_{e c r}=H_{L A D}(q)\left(h_{I R C, e c r}\left(u_{e c r}\right)+d_{e c r}\right)$ is produced by the ECR muscle. Finally, let each moment feed into the linear wrist rigid body dynamics, $H(q)$, to produce joint angle $y=H(q)\left(\tau_{f c r}-\tau_{e c r}\right)$. For both muscles $u_{f c r}, u_{e c r} \geq 0$, and the monotonically increasing recruitment curves satisfy $h_{f c r}(0)=h_{\text {ecr }}(0)=0$.

An additional component can be added at the beginning of the model in Fig. 1 to mimic natural muscle coactivation, and it is here modelled by a function relating the FES signals to a single control input, defined by $\left(u_{f c r}(k), u_{e c r}(k)\right)^{\top}=$ $h_{C C}(u(k))$ where

$$
\begin{aligned}
& u_{f c r}(k)=\left\{\begin{array}{ll}
u(k)+u_{c, f c r} & u(k) \in\left[0,300-u_{c, f c r}\right] \\
u_{c, f c r} & u(k) \in\left[u_{c, e c r}-300,0\right]
\end{array} ;\right. \\
& u_{e c r}(k)= \begin{cases}u_{c, e c r} & u(k) \in\left[0,300-u_{c, f c r}\right] \\
u_{c, e c r}-u(k) & u(k) \in\left[u_{c, e c r}-300,0\right]\end{cases}
\end{aligned}
$$

with co-activation levels $u_{c, f c r}, u_{c, e c r} \in \mathbb{R}_{+}$. Here a maximum pulsewidth of $300 \mu \mathrm{s}$ is assumed, based on that used in commercial stimulators, and the range known to provide comfortable contraction [31]. This modulates mechanical impedance to maintain postural stability and is the method in which the central nervous system naturally activates antagonistic muscles during task performance [32], [33]. 


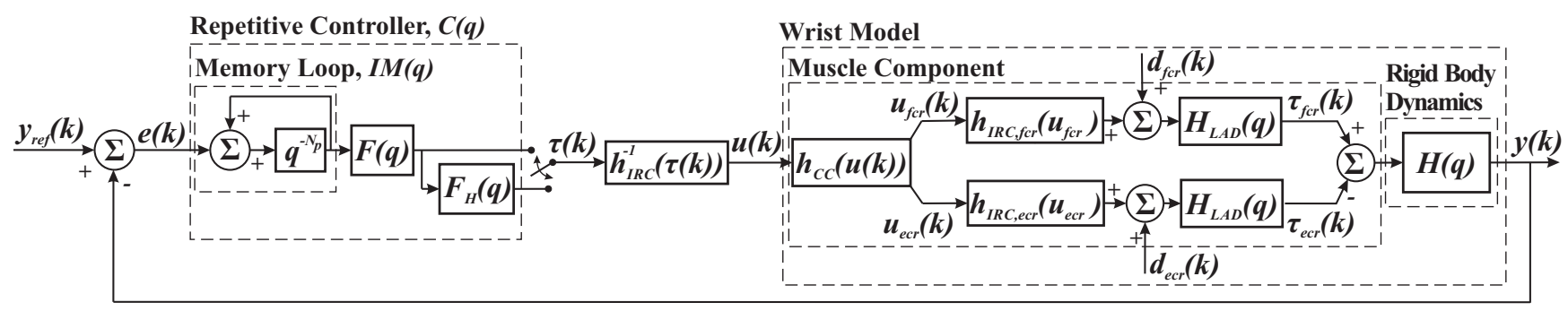

Fig. 1. Closed-loop control system structure in which the wrist model of Definition 1 is excited by electrical stimulation and tremor.

The following proposition addresses selection of coactivation parameters and will be needed in later control development.

Proposition 1: Let the co-activation levels be chosen to satisfy $h_{I R C, f c r}\left(u_{c, f c r}\right)=h_{I R C, e c r}\left(u_{c, e c r}\right)$. Then the function

$$
h_{I R C}(u):=h_{I R C, f c r}\left(u_{f c r}\right)-h_{I R C, e c r}\left(u_{e c r}\right)
$$

is continuous and monotonic increasing over the domain $u(k) \in\left[u_{c, e c r}-300,300-u_{c, f c r}\right]$, with $h_{I R C}(0)=0$.

Proof. Inserting (1) into (2) gives $h_{I R C}(u)=$

$$
\begin{cases}h_{I R C, f c r}\left(u+u_{c, f c r}\right)-h_{I R C, e c r}\left(u_{c, e c r}\right), & u \geq 0 \\ -h_{I R C, e c r}\left(u_{c, e c r}-u\right)+h_{I R C, f c r}\left(u_{c, f c r}\right), & \text { otherwise }\end{cases}
$$

From Definition $1, h_{I R C, f c r}(\cdot)$ and $h_{I R C, e c r}(\cdot)$ are continuous and monotonically increasing over their domain $[0,300]$. Hence it follows that the domain of $h_{I R C}(u)$ is $\left[u_{\min }, u_{\max }\right]$, where $u_{\min }=u_{c, e c r}-300$ and $u_{\max }=300-u_{c, f c r}$. Moreover, since $h_{I R C, f c r}\left(u_{c, f c r}\right)=h_{I R C, e c r}\left(u_{c, e c r}\right), h_{I R C}(u)$ is continuous, monotonic and satisfies $h_{I R C}(0)=0$

The identification problem can then be stated as follows:

Definition 2 (Identification Problem): Consider the stimulated wrist system defined by Definition 1 , and let its constituent components be explicitly parametrized by $\theta$. Given a set of sampled input-output data $\{u(k), y(k)\}_{k=1, \cdots, N}$, the identification problem is to find optimal parameter estimate

$$
\hat{\theta}=\underset{\theta}{\arg \min } \sqrt{\sum_{k=1}^{N}(y(k)-\hat{y}(k \mid k-1))^{2}}
$$

where $\hat{y}(k \mid k-1)$ is the one-step-ahead predicted output associated with the assumed model description

$$
\begin{aligned}
\hat{y}= & H(q, \theta) H_{L A D}(q, \theta)\left\{\left[d_{f c r}(\theta)+h_{I R C, f c r}\left(u_{f c r}, \theta\right)\right]\right. \\
& \left.-\left[d_{e c r}(\theta)+h_{I R C, e c r}\left(u_{e c r}, \theta\right)\right]\right\}+\nu
\end{aligned}
$$

with a zero mean disturbance $\nu$.

The identification problem can be solved by assuming the following component forms which are motivated by the model set-up and coactivation parameter selection of Proposition 1.

Theorem 1: Let the components within the wrist model defined by Definition 1 be represented by the general forms:

$$
\begin{array}{r}
H(q, \theta) H_{L A D}(q, \theta):=P(q, \theta) \\
=\frac{b_{0} q^{-m}+b_{1} q^{-(m+1)}+\cdots+b_{n} q^{-(m+n)}}{1+a_{1} q^{-1}+\cdots+a_{l} q^{-l}},
\end{array}
$$

$$
\begin{array}{r}
h_{I R C, f c r}\left(u_{f c r}, \theta\right)-h_{I R C, e c r}\left(u_{e c r}, \theta\right)=h_{I R C}(u, \theta) \\
=\mu_{0}+\mu_{1} u+\cdots+\mu_{s-1} u^{s-1}+\mu_{s} u^{s},
\end{array}
$$

with recruitment curve monotonicity conditions embedded as

$$
\left.\frac{d h_{I R C}}{d u}\right|_{\substack{u=u_{0} \\=\mu_{1}}}+\ldots+\mu_{s-1}(s-1) u^{s-2}+\mu_{s} s u^{s-1} \geq 0
$$

and the general $N_{p}$-period tremor description

$$
\begin{aligned}
d_{f c r}(\theta) & -d_{e c r}(\theta):=d(\theta) \\
& =A_{0}+\sum_{i=1}^{N_{f}}\left\{A_{i} \cos \left(\frac{2 \pi i k}{N_{p}}\right)+B_{i} \sin \left(\frac{2 \pi i k}{N_{p}}\right)\right\}
\end{aligned}
$$

where $N_{f}$ is the highest harmonic. Then the identification problem of Definition 2 is solved by the iterative algorithm defined in [20] to produce the optimal parameter vector

$$
\theta=\left[a_{1} \cdots a_{l}, b_{0} \cdots b_{n}, A_{0} \cdots A_{N_{f}}, B_{1} \cdots B_{N_{f}}, \mu_{0} \cdots \mu_{s}\right]^{\top}
$$

Proof. See [20].

Remark 1: The model and identification forms can be extended to capture the case in which the tremulous muscle activity affects the recruitment characteristics of the FCR and ECR muscles during stimulation. In this case the recruitment function will have form $\hat{h}_{I R C}(u, \delta)$ where $\delta$ is the $N_{p}$-periodic signal formed by unknown tremulous signals $\delta_{f c r}$ and $\delta_{\text {erc }}$ [34]. The periodic form means that, without loss of generality,

$$
\hat{h}_{I R C}(u, \delta, \theta)=\tilde{h}_{I R C}(u, \theta, k)+\tilde{h}_{I R C}(\delta), \quad k=1, \ldots, N
$$

where $\tilde{h}_{I R C}(u, \theta, k):=h_{I R C}(u, \theta(i(k)))$, where $i$ is the period index of sample $k$, and the extended parameter vector

$$
\begin{aligned}
\theta(i(k))=\left[a_{1} \cdots a_{l}, b_{0} \cdots b_{n}, A_{0} \cdots A_{N_{f}}, B_{1} \cdots B_{N_{f}},\right. \\
\left.\mu_{0}(i) \cdots \mu_{s}(i)\right]^{\top}, \quad i=1, \ldots N_{p} .
\end{aligned}
$$

Hence $h_{I R C}(u, \theta(i(k)))$ and $\theta(i(k))$ replace (7) and (10) respectively in Theorem 1 , with $N_{p}$-periodic term $\tilde{h}_{I R C}(\delta)$ absorbed into $d(\theta)$. Theorem 1 then only requires a minor extension of the identification procedure of [20] to hold for this more general characterisation of tremor. This is easily managed by partitioning data sets, but inevitably will require longer identification tests. Note that the controller proposed in Section III is unchanged (but $h_{I R C}^{-1}(\cdot)$ will be time-varying).

Having defined and identified the system from experimental data, the control strategy is developed in the next section. 


\section{TREMOR SUPPRESSION USING RC}

The control strategy introduced next combines linearising control action with RC, in which design of the latter considerably simplified through application of the former.

Theorem 2: Consider the repetitive control action

$$
u=h_{I R C}^{-1}\left(F(q)\left(q^{N_{p}}-1\right)^{-1} e\right)
$$

with $F(q)$ taking the form

$$
\begin{aligned}
F(q)= & \kappa_{1} q^{\beta-1}+\kappa_{2} q^{\beta-2}+\cdots+\kappa_{\beta} q^{0}+\cdots \\
& +\kappa_{\alpha-1} q^{-(\alpha-\beta-1)}+\kappa_{\alpha} q^{-(\alpha-\beta)}
\end{aligned}
$$

where $\beta \leq N_{p}+1$, applied to the system of Definition 1 with components satisfying Proposition 1 and Theorem 1 . Then $N_{p^{-}}$ periodic disturbance $d$ is completely rejected if and only if transfer function $F(q)$ is chosen such that the roots of

$$
1-q^{-N_{p}}(1-P(q) F(q))=0
$$

are all inside the unit circle. A sufficient condition is given by

$$
\|1-P(q) F(q)\|_{\infty}<1
$$

or equivalently, in the frequency domain by

$$
\sup _{\omega \in[0, \pi]}\left|1-P\left(e^{j \omega}\right) F\left(e^{j \omega}\right)\right|<1,
$$

assuming that $1-P(q) F(q)$ is internally stable.

Proof. The identified forms (7), (8) guarantee that $h_{I R C}(u)$ is monotonically increasing and hence has an inverse $h_{I R C}^{-1}(\tau)$ defined over $\left[u_{\min }, u_{\max }\right]$. Having applied this function, the standard linear repetitive control system with form

$$
\tau\left(k+N_{p}\right)=\tau(k)+F(q) e(k)
$$

generates the control action (11). This has a sufficient condition for complete disturbance rejection given by (14), as shown in [35] or condition (15) as shown in [36], [37].

The tremulous wrist model of Definition 1 and control action of Theorem 2 result in the system shown in Fig. 1. In the necessary condition for monotonic convergence (15), the term $\left|1-P\left(e^{j \omega}\right) F\left(e^{j \omega}\right)\right|$ dictates how rapidly frequency component $\omega \in[0, \pi]$ of the error diminishes to zero. Hence to obtain the fastest learning rate, $F(q)$ should be selected as the right inverse of the linear dynamics $P(q)$. However, this is often infeasible due to instability that occurs when the true plant differs from $P(q)$ due to the presence of effects such as fatigue and spastacity [37].

In practice, choosing $F(q)$ to satisfy (14) or (15) is challenging, especially if the system has zeros outside the unit circle. The following methods, namely frequency modified inverse RC (FMI-RC) and gradient based RC (GB-RC), have been selected from the many available $\mathrm{RC}$ variants because of their track record in experimental effectiveness, attractive robustness properties, and ease of implementation [35], [37].

\section{1) Frequency Modified Inverse RC (FMI-RC):}

Algorithm 1: Condition (15) can be addressed by selecting $F(q)$ as an approximation of $P^{-1}(q)$ through suitable choice of parameters $\phi=\left[\begin{array}{llll}\kappa_{1} & \kappa_{2} & \cdots & \kappa_{\alpha}\end{array}\right]^{\top}$ appearing in $F(q)$ definition (12). This is achieved by minimising the cost function

$$
J=\sum_{i=0}^{L}\left[1-P\left(e^{j \omega_{i}}\right) F\left(e^{j \omega_{i}}\right)\right]\left[1-P\left(e^{j \omega_{i}}\right) F\left(e^{j \omega_{i}}\right)\right]^{*}
$$

where $\omega_{i}=2 \pi f_{i} T$ in which $\left\{f_{i}\right\}_{i=0, \ldots, L}$ is an appropriate set of frequencies selected from 0 to $f_{N}=1 / 2 T$.

The solution, $\phi^{*}$, to (17) is given by $\phi^{*}=\Gamma^{-1} \Phi$ where

$$
\begin{aligned}
\Gamma & =\sum_{i=0}^{L} M^{2}\left(\omega_{i}\right) \Psi_{i}, \\
\Phi & =\sum_{i=0}^{L} M\left(\omega_{i}\right)\left[\begin{array}{c}
\cos \left((\beta-1) \omega_{i}+\varphi\left(\omega_{i}\right)\right) \\
\vdots \\
\cos \left((\beta-\alpha) \omega_{i}+\varphi\left(\omega_{i}\right)\right)
\end{array}\right]
\end{aligned}
$$

in which $\Psi_{i} \in R^{\alpha \times \alpha}$ is

$$
\Psi_{i}=\left[\begin{array}{cccc}
1 & \cos \left(\omega_{i}\right) & \cdots & \cos \left((\alpha-1) \omega_{i}\right) \\
\cos \left(\omega_{i}\right) & 1 & \cdots & \cos \left((\alpha-2) \omega_{i}\right) \\
\vdots & \vdots & \ddots & \vdots \\
\cos \left((\alpha-1) \omega_{i}\right) & \cos \left((\alpha-2) \omega_{i}\right) & \cdots & 1
\end{array}\right] \text { (19) }
$$

and $M(\omega)$ and $\varphi(\omega)$ are the magnitude and phase of $P(q)$, respectively. It is further shown in [38] that computation of $\phi^{*}$ over a suitably wide range of $\alpha$ and $\beta$ values, together with introduction of a frequency weighting to (17) can be utilised to increase learning over specified frequency ranges. This effectively enables the designer to transparently manipulate selection of $F(q)$ in the frequency domain in order to compute an approximation of the inverse of $P(q)$ which attempts to satisfy (15), however no guarantee of success is possible.

\section{2) Gradient-based $R C(G B-R C)$ :}

Algorithm 2: Condition (15) is satisfied by choosing $\alpha=$ $\beta=N_{p}$ in (12), together with

$$
\kappa_{i}=\gamma h_{N_{p}-i}, \quad i=1, \ldots, N_{p},
$$

in which $h_{i}$ are the Markov parameters of $P(q)$ with $h_{i}=0$ $\forall i>N_{p}$, and $\gamma$ selected as

$$
0<\gamma<\frac{2}{\sup _{\omega \in[0, \pi]}\left|P\left(e^{j \omega}\right)\right|^{2}} .
$$

To see this note that the choice (20) is equivalent to setting

$$
\begin{aligned}
F(q)=\sum_{i=1}^{N_{p}} \kappa_{i} q^{N_{p}-i} & =\gamma \sum_{i=1}^{N_{p}} h_{N_{p}-i} q^{N_{p}-i} \\
& =\gamma P\left(q^{-1}\right)=\gamma P^{*}(q) .
\end{aligned}
$$

Substituting (22) into the left-hand side of (15) yields

$$
\begin{aligned}
\sup _{\omega \in[0, \pi]}\left|1-P\left(e^{j \omega}\right) F\left(e^{j \omega}\right)\right| & =\sup _{\omega \in[0, \pi]}\left|1-\gamma P\left(e^{j \omega}\right) P^{*}\left(e^{j \omega}\right)\right| \\
& =\left.\sup _{\omega \in[0, \pi]}|1-\gamma| P\left(e^{j \omega}\right)\right|^{2} \mid
\end{aligned}
$$


and it follows that selection (21) satisfies (15). The gradient based RC update is described in [35], [39] which establish its attractive robustness and convergence properties.

Remark 2: The assumption in Algorithm 2 that $h_{i}=0 \forall i>$ $N_{p}$ can be addressed through the introduction of appropriate feedback action, e.g. deadbeat control, pole-placement. Plant $P(q)$ is then replaced by the resulting closed-loop structure. Note that evaluating (22) approximates a plant inverse at low frequencies, but the learning rate reduces for higher frequencies. This embeds robustness to plant uncertainty into the RC structure, which is discussed further in [35].

\section{INTERFERENCE of RC With Voluntary Motion}

Section III proposed a control strategy for complete tremor suppression at the wrist. However it is possible that the control action of Theorem 2 may interfere with patients' ability to extend or flex their wrist voluntarily, and thereby impair their performance of functional activities. To examine whether the proposed RC scheme has any undesirable effect on voluntary motion, the following assumptions are necessary:

A1. Voluntary wrist movement occurs at frequencies less than $1 \mathrm{~Hz}$, as shown in [40].

A2. Involuntary movement associated with intention tremor occurs at a single frequency between $2 \mathrm{~Hz}$ and $5 \mathrm{~Hz}$, as shown in [1], [2].

A3. Voluntary action manifests as an additive signal, $v$, appearing in the summation blocks within Fig. 1 that preceed $H_{L A D}$. This is consistent with the underlying mechanism assumed to produce the tremor signal $d$.

\section{A. Distortion of voluntary intention}

Suppose the control action of Theorem 2 is applied to suppress tremor while voluntary action $v$ is also exerted by the patient. Then the distortion in the intended movement is

$$
\Delta y_{v}=\left\|y_{v}-y\right\|_{2}
$$

where $y_{v}=P(q) v$ and $y=S(q) v$ with

$$
S(q)=P(q)\left(1+P(q) F(q)\left(q^{N_{p}}-1\right)^{-1}\right)^{-1} .
$$

It follows that to minimise the distortion (23), a necessary and sufficient condition is that the control action of Theorem 2 must satisfy

$$
\left|S\left(e^{j \omega}\right)\right|=\left|P\left(e^{j \omega}\right)\right|, \text { and } \angle S\left(e^{j \omega}\right)=\angle P\left(e^{j \omega}\right)
$$

for frequencies less than $1 \mathrm{~Hz}$.

Theorem 3: It is impossible for any $F(q)$ satisfying either (14) or (15) to satisfy either condition (25). It follows that there exists $v$ such that $\Delta y_{v} \neq 0$.

Proof. Equation (24) can be rewritten as

$$
S(q)=P(q) \frac{q^{N_{p}}-1}{q^{N_{p}}-(1-P(q) F(q))} .
$$

It is then obvious that $\left|S\left(e^{j \omega}\right)\right|$ approaches 0 as $\omega \rightarrow 0$ for all $F(q)$ satisfying either (14) or (15), and hence never satisfies (25) since $\lim _{\omega \rightarrow 0}\left|P\left(e^{j \omega}\right)\right| \neq 0$.

\section{B. Distortion elimination}

This subsection proposes a method to address the interference of the controller with the voluntary movement that is established in Theorem 3. This is achieved by adding a ZPHP filter, $F_{H}(q)$, in series with the repetitive control action. The placement of $F_{H}(q)$ is shown in Fig. 1, and its implementation requires replacing $C(q)=F(q)\left(q^{N_{p}}-1\right)^{-1}$ with the more general expression

$$
C(q)=F_{H}(q) F(q)\left(q^{N_{p}}-1\right)^{-1} .
$$

The aim is to shape the frequency response of the closedloop system at low frequencies in order to satisfy (25), while preserving tremor suppression at higher frequencies.

Theorem 4: Suppose the control action of Theorem 2 is implemented with $F(q)$ satisfying either (14) or (15). Then suppose an ideal, non-causal ZPHP filter, $F_{H}(q)$, with cut-off frequency $\omega_{c}$ and characteristic

$$
\begin{aligned}
& \left|F_{H}(\omega)\right|=\left\{\begin{array}{ll}
0, & \omega \in\left[0, \omega_{c}\right] \\
1, & \omega \in\left(\omega_{c}, \pi\right]
\end{array},\right. \\
& \angle F_{H}(\omega)=0 \quad \forall \omega \in\left(\omega_{c}, \pi\right]
\end{aligned}
$$

is added immediately following $F(q)$, as shown in Fig. 1. Then (25) is satisfied for $\omega \in\left[0, \omega_{c}\right]$, thereby eliminating voluntary distortion. In addition, $\omega \in\left(\omega_{c}, \pi\right]$ frequency components of $y$ asymptotically converge to patients' intended motions $y_{r e f}$, thereby eliminating tremor.

Proof. Adding $F_{H}(q)$ means (26) is replaced by

$$
S(q):=P(q) V(q)
$$

where $V(q)=(1+P(q) C(q))^{-1}$ with $C(q)$ given by (27). Due to the frequency response characteristic (28), the frequency response of $V(q)$ is

$$
\begin{aligned}
& \left|V\left(e^{j \omega}\right)\right|=\left|\frac{q^{N_{p}}-1}{q^{N_{p}}-\left(1-P(q) F(q) F_{H}(q)\right)}\right|_{q=e^{j \omega}}=1, \\
& \angle V\left(e^{j \omega}\right)=\left.\angle\left(\frac{q^{N_{p}}-1}{q^{N_{p}}-\left(1-P(q) F(q) F_{H}(q)\right)}\right)\right|_{q=e^{j \omega}}=0,
\end{aligned}
$$

$\forall \omega \in\left[0, \omega_{c}\right]$, so that the magnitude and phase responses of (29) equate to $|P(q)|$ and $\angle P(q)$, respectively. It follows that an ideal ZPHP filter satisfies (25) for $\omega=\left[0, \omega_{c}\right]$. Replacing $F(q)$ by $F(q) F_{H}(q)$ in (15) results in

$\left|1-F\left(e^{j \omega}\right) F_{H}\left(e^{j \omega}\right) P\left(e^{j \omega}\right)\right| \leq\left|1-F\left(e^{j \omega}\right) P\left(e^{j \omega}\right)\right|<1, \forall \omega \in\left(\omega_{c}, \pi\right]$

since $\angle F_{H}\left(e^{j \omega}\right)=0$ and $\left|F_{H}\left(e^{j \omega}\right)\right|=1, \forall \omega \in\left(\omega_{c}, \pi\right]$. Hence the RC convergence criterion for the augmented system is satisfied over the involuntary frequency range.

Remark 3: Since an ideal ZPHP filter requires an infinite amount of data to obtain the desired characteristic (28), it must be realised instead by the finite length approximation

$F_{H}(q)=f_{0} q^{-N_{H}}+f_{1} q^{-N_{H}+1}+\cdots+f_{N_{H}}+f_{N_{H}+1} q+\cdots+f_{2 N_{H}} q^{N_{H}}$ with a cut-off frequency $\omega_{c}$, in which $2 N_{H}+1$ is the filter length. This must be chosen to satisfy $N_{H} \leq N_{p}-\beta+1$ to ensure that control action $C(q)$, given by (27), is proper and therefore causal.

The next subsection illustrates how to apply Theorem 4 using the specific RC designs of Section III. 


\section{The application of Theorem 4 to $R C$ design}

Remark 3 indicates that filter parameter $N_{H}$ must be suitably large to realise characteristics (28) within Theorem 4. However this restricts the parameter $\beta$ needed to satisfy (14) and (15) within the same theorem. This section provides guidelines for satisfying Theorem 4 for the case of the two $\mathrm{RC}$ approaches considered previously, enabling the designer to balance the competing objectives.

For Algorithm 1 of FMI-RC, $\beta$ is prescribed by the designer. For the system forms typically encountered in the present application, reducing $\beta$ makes it increasingly difficult to produce a suitable inverse which satisfies (14) and (15). However, this observation does not hold for all system types.

For Algorithm 2 of GB-RC, $\beta$ is dictated by the impulse response length of $P(q)$. Decreasing $\beta$ causes the assumption in Algorithm 2 to be replaced by $h_{i}=0 \forall i>\beta$ and results in increasing difficulty to fulfill the assumption. However by applying Remark 2 , this effect can be reduced by the introduction of an appropriate feedback action.

\section{Effect of non-ideal ZPHP filter on convergence}

The restriction on $N_{H}$ highlighted in Remark 3 means that the realised ZPHP filter $F_{H}(\omega)$ will have ripples in its magnitude response within the passband $\forall \omega \in\left(\omega_{c}, \pi\right]$. To investigate the effect of this deviation in unity magnitude, $F_{H}(\omega)$ can be introduced into convergence criteria (15) and routine analysis applied to yield sufficient convergence condition

$$
\left|F_{H}(\omega)\right|<\frac{2 \cos \left(\angle\left(P\left(e^{j \omega}\right) F\left(e^{j \omega}\right)\right)\right)}{\left|P\left(e^{j \omega}\right) F\left(e^{j \omega}\right)\right|} \quad \forall \omega \in\left(\omega_{c}, \pi\right] .
$$

Since (15) holds, the right side of (32) can be shown to satisfy

$$
\frac{2 \cos \left(\angle\left(P\left(e^{j \omega}\right) F\left(e^{j \omega}\right)\right)\right)}{\left|P\left(e^{j \omega}\right) F\left(e^{j \omega}\right)\right|}>1 \quad \forall \omega \in\left(\omega_{c}, \pi\right] .
$$

Design of $F$ can therefore transparently manipulate bound (32) and can guarantee it holds for any ripple magnitude. For example, in the case of GB-RC (32) becomes

$$
\left|F_{H}(\omega)\right|<\frac{2}{\gamma\left|P\left(e^{j \omega}\right)\right|^{2}} \quad \forall \omega \in\left(\omega_{c}, \pi\right]
$$

and $\gamma$ can always be reduced to ensure it is satisfied.

Remark 4: This section has proposed a method in which low frequency voluntary motion may be preserved, while high frequency tremor is suppressed. Within the RC structure, this separation can be achieved with zero phase distortion, however in common with all frequency-based designs, voluntary motion is inevitably affected if the frequency ranges overlap. The structure of RC can address this problem by modifying the current internal model (i.e. $\operatorname{IM}(q)=\left(q^{N_{p}}-1\right)^{-1}$ ) so that it only learns periodic signals that are known to correspond to tremulous motion (rather than all periodic signals, as is currently the case). A simple example is an $N_{p}$-periodic sinewave (in which case $\left.I M(q)=\left(q^{3}-2.996 q^{2}+2.996 q-1\right)^{-1}\right)$, but any form of signal (or groups of signal) can be incorporated in a straightforward manner [41]. Using this modified internal model with the remaining design procedure unchanged thereby minimises the controller's interference with voluntary motion above $\omega_{c}$, while still ensuring complete tremor suppression.

\section{Experimental Test Procedure}

A systematic experimental approach has been designed to investigate the feasibility of the FES based tremor suppression system using RC. Ethical approval was obtained from the University of Southampton (ERGO Ref: 16530). In Phase 1 of the study four unimpaired people were recruited, each satisfying the following criteria: (i) no motor dysfunction or diagnosed systemic conditions; (ii) no pacemakers/attached electronic equipment; (iii) no cognitive/visual impairments; (iv) no diagnosed skin disorder; (v) able to provide informed consent. In Phase 2 a single person with MS (PwMS) was recruited using the same criteria, together with: (vi) a stable presentation of intention tremor primarily affecting the distal joints. All participants gave written informed consent.

\section{A. Testing apparatus}

1) Wrist Rig: A wrist rig was employed that has been specifically designed and validated to detect changes in upper limb function [42]. The participant's arm was supported by two Velcro straps wrapped around the top and bottom of the elbow, and by a foam splint supporting the forearm, as shown in Fig. 2. This prevented any confounding movement of the arm so all movements were limited to the wrist joint. The rig was designed to present tracking tasks to patients and assess their performance in completing them. To do this, it includes 80 LED lights spaced at $2^{\circ}$ increments measuring an arc of wrist joint movement from $80^{\circ}$ flexion to $80^{\circ}$ extension. The angle of the wrist, $y$ was measured by a potentiometer aligned with wrist flexion/extension and was recorded by realtime hardware (dSpace 1103). Then analysis was conducted in MATLAB. The real-time hardware also implemented the proposed control scheme which generated FES signals, $u_{f c r}$ and $u_{e c r}$. These comprised 5V pulse width modulation (PWM) sequences which were fed to the commercial FES device.
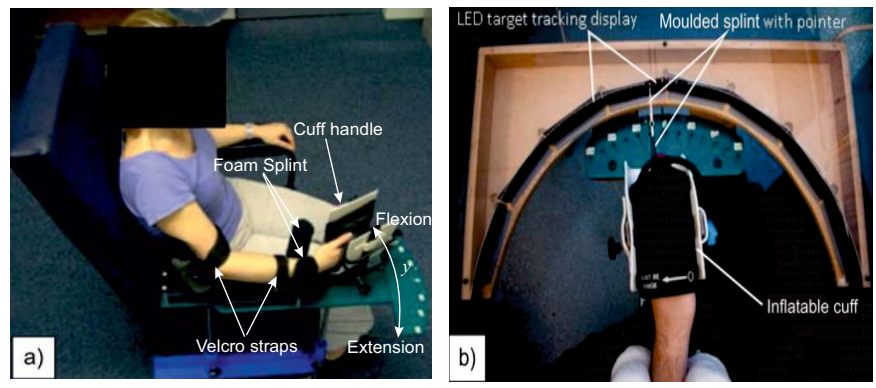

Fig. 2. Experimental set-up (permission obtained to reproduce [42]): a) the participant's arm supported by the wrist rig, b) an overhead view of the wrist.

2) FES Device: The output stage of a voltage-controlled Odstock $^{\circledR}$ 4-channel neuromuscular stimulator was used to amplify PWM pulse trains to form $40 \mathrm{~Hz}$ bi-phasic waveforms.

3) Induced Tremor: As the Phase 1 feasibility tests were conducted with unimpaired participants, it was necessary to induce tremor artificially at realistic frequencies. This was done by modifying the wrist rig to include a mechanical system driven by a geared DC motor. This system consisted of three units, a brushed DC motor (Beijing V.T.V) equipped with a gearbox of 5:1 gear ratio and $1.28 \mathrm{Nm}$ power at $360 \mathrm{rpm}$, 
a DC power supply to drive the motor and a motor controller (Maxon Motor ESCON). The motor torque was transmitted through a shaft to the link connected with the molded splint on which participants placed their wrist and hand as shown in Fig. 3. Thus the wrist and hand oscillated when the motor applied torque at the desired frequency. The motor controller ensured the torque was independent of speed in order to match the model assumption of Definition 1 that tremulous motion manifests as the involuntary muscle torque $d$. The motor was disconnected during the subsequent Phase 2 PwMS tests.

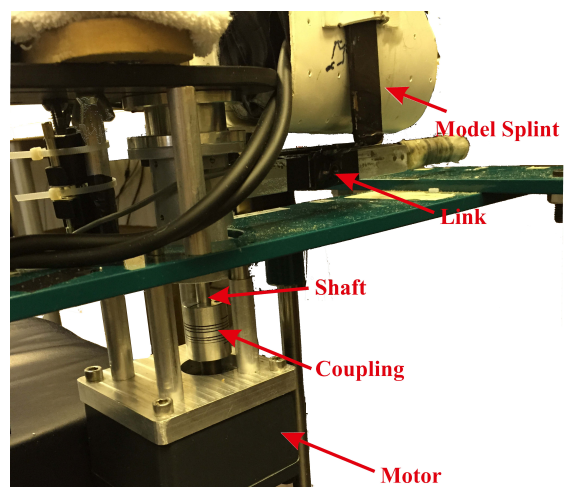

Fig. 3. The electromechanical system used to induce tremor artificially.

\section{B. Data Collection Procedure}

1) Test Setup: Participants placed their right arm into the wrist rig's adjustable padded armrest. The length of the armrest was adjusted to allow the wrist joint to aligned directly over the potentiometer so the wrist joint angle was accurately measured. Using standard guidelines [43], two sets of PALS Plus adhesive $5 \times 5 \mathrm{~cm}$ surface electrodes were placed on the forearm to stimulate the FCR and ECR muscles and connected to the corresponding outputs of the Odstock ${ }^{\circledR}$ stimulator. The amplitude level of each channel was individually set to a maximum value which did not cause discomfort to participants while the maximal pulse width $(300 \mu \mathrm{s})$ was applied.

2) Control Design/Parameter Selection: The sampling frequency was set to $200 \mathrm{~Hz}(T=0.005 \mathrm{~s})$. During the Phase 1 tests an artificial tremor of frequency $f_{p}=2 \mathrm{~Hz}\left(N_{p}=100\right)$ was induced using the mechanical system. This frequency was chosen since the power spectrum of EMG signals measured from patients with intention tremor typically shows that tremor has a dominant frequency between $2-5 \mathrm{~Hz}$ [44].

a) Identification of wrist model: In the case of Phase 2 tests the tremor frequency was first identified via Discrete Fourier Transform (DFT). In order to identify the wrist model using Theorem 1, an input $u$ comprising a set of sine waves that spanned the frequency range from $0.2 \mathrm{~Hz}$ to $4 \mathrm{~Hz}$ was applied to both muscle groups and the angular position $y$ of the wrist joint was acquired. Following Theorem 1, the input/output data were then used to identify the model components $P(q)$ and $h_{I R C}$. To quantify accuracy, a validation test was conducted in which the set of input sequences was reapplied and the model used to predict the response. b) $R C$ control design: Having obtained the model, the control approach of Theorem 4 was applied. To suppress tremor, $y_{r e f}$ was chosen to be 0 . First, RC was designed without a ZPHP filter. In this case, $\beta \leq N_{p}=100$ was selected and RC forms were designed to satisfy either (14) or (15). Then the ZPHP filter $F_{H}(q)$ of Theorem 4 was designed using $N_{H}=50$ and a cut-off frequency of $\omega_{c}=1.2 \mathrm{~Hz}$, following the guidelines of Remark 3. RC forms were modified with the more restrictive condition $N_{H} \leq N_{p}-\beta+1=51$. Thus Theorem 4 was satisfied for both RC Algorithms 1 and 2. The coefficients of $F_{H}(q)$ were obtained from the convolution of the impulse response of a $6^{\text {th }}$ order high-pass Butterworth filter with itself. The frequency response of the ZPHP filter is shown in Fig. 4, which approximates the desired response given by (28) within Theorem 4.

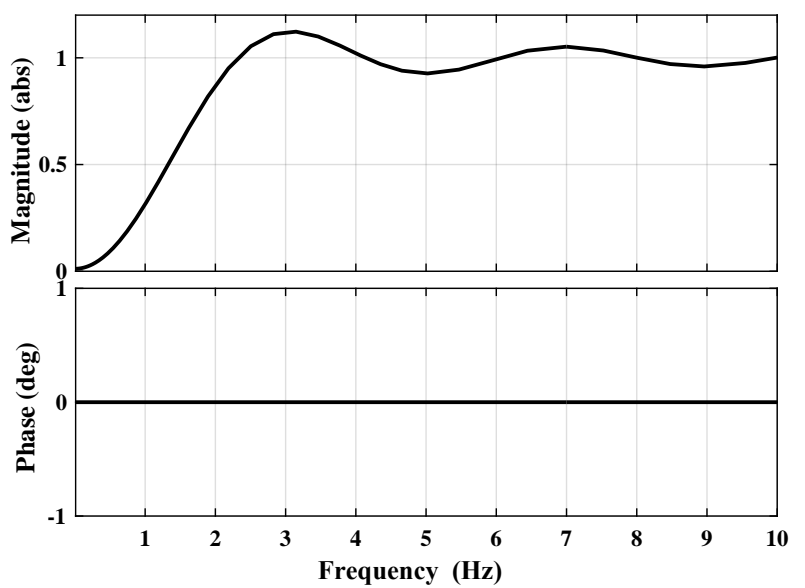

Fig. 4. Bode plot of the ZPHP filter used experimentally.

To illustrate the design procedure, frequency responses of the relationship between $v$ and $y$ are shown in Fig. 5 with and without the ZPHP filter $F_{H}(q)$ (corresponding to (26) and (29) respectively). Comparison with $P(q)$ reveals that RC without employing the ZPHP filter has a marked interference with voluntary motion. This interference would cause RC to provide high stiffness in the low frequency region. Therefore, participants may be expected to have difficulty in moving their wrist, thereby impairing wrist function. In contrast, it is apparent from Fig. 5 that employing the ZPHP filter in RC can satisfy (25), thereby leading to minimal interference with voluntary motion at frequencies less than $1 \mathrm{~Hz}$.

c) Conventional filter design: The proposed control structures were compared with the leading conventional filtering technique comprising a high-pass filter (HPF) placed in the feedback path. Design requirements were chosen to correspond to those previously employed for tremor suppression [18]. In particular, a $6^{t h}$ order Butterworth-type HPF was implemented due to its flat magnitude response, with an order and cut-off frequency chosen to guarantee a maximum closed-loop gain in the range of $2-5 \mathrm{~Hz}$ (to suppress tremor) and a minimum gain and phase lag in the $0-1 \mathrm{~Hz}$ range (to preserve voluntary action).

3) Data Collection: In Phase 1 of the study the following tests were performed to fully evaluate the control approaches 


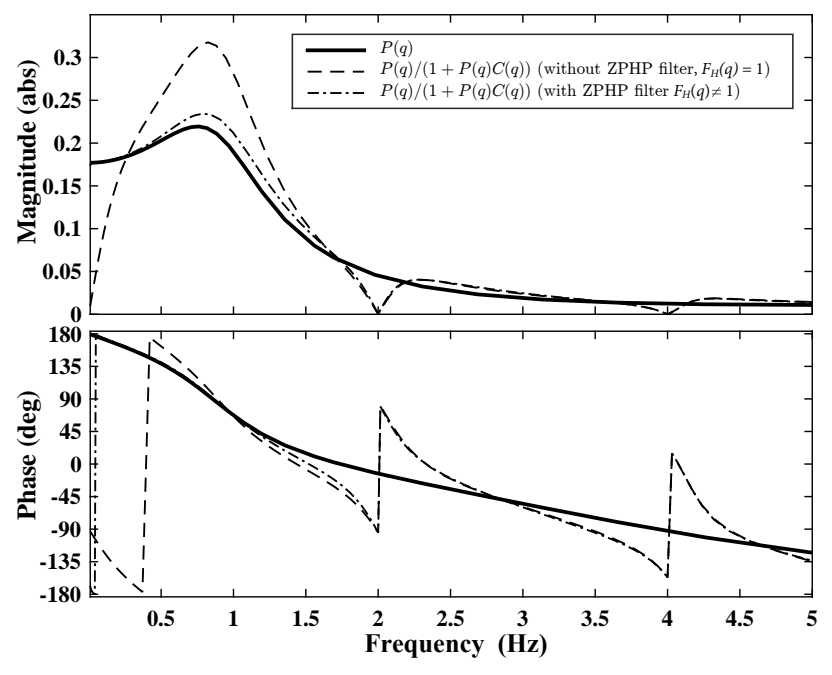

Fig. 5. Bode plots of $P(q)$ and the closed-loop relationship $S(q)$.

(with and without the ZPHP filter):

T1. Tracking task with neither induced tremor nor FES

T2. Tracking task with induced tremor but without FES

T3. Tracking task with induced tremor and FES

Tracking Test $T 1$ comprised a step-tracking task [42], in which participants were instructed to flex or extend their wrist to track the position indicated by the LEDs which were illuminated in a pseudo-random pattern. Each LED was lit for 6 seconds and the test lasted for 40 seconds. Tracking Test $T 2$ examined the effect of induced tremor on participant's ability to perform the same tracking task. Tracking Test $T 3$ examined the capability of FES to suppress the induced tremor while preserving participants' ability to perform the voluntary task.

FES recruits fast-twitch muscle fibres before slow-twitch fibres which increases the rate of fatigue [45], therefore a rest of 5 min took place after each test, a time frame recommended for patients with disability [4]. A training session was carried out for each participant on the day prior to testing in order to familiarise them with the test procedure and allow them to become accustomed to FES. In this session participants undertook Tracking Tests $T 1$ and $T 2$ at least 3 times, with a rest period of 2 min between each test. This enabled them to practice tracking reference paths in both tremor scenarios (i.e. where induced tremor was absent and present). Then the stimulation inputs used in the identification procedure were applied to their relevant muscles to get them used to FES. On the following day, the test session took place and the aforementioned tests were performed. Due to the many tests, each was performed only once, but control parameters were varied to fully explore achievable performance.

In Phase 2 of the study, the test procedure was identical except that Test $T 1$ was omitted and no tremor was induced.

4) Data Analysis: To quantify both tremor suppression and preservation of voluntary intention during tests $T 2$ and $T 3$, their outputs, $y$ must be compared with the ideal response to the tracking task (termed $y_{v}$ ). In Phase 1 this was provided by Test $T 1$, and in Phase 2 an average of the Phase $1 y_{v}$ signals was used (since Test $T l$ cannot be performed with PwMS).
Then the norm of the output over the involuntary frequency range is used to quantify tremor suppression, and is denoted by

$$
\Delta y_{t}=\left\|\left.\left(y_{v}-y\right)\right|_{\left[\omega_{c}, \pi\right]}\right\|_{2}=\left\|\left.y\right|_{\left(\omega_{c}, \pi\right]}\right\|_{2} .
$$

which follows from Assumption A.1 since $y_{v}$ is zero over the frequency range $\omega \in\left(\omega_{c}, \pi\right]$. The following norm over the voluntary frequency response was used to quantify the preservation of voluntary intention, and is denoted by

$$
\Delta y_{v}=\left\|\left.\left(y_{v}-y\right)\right|_{\left[0, \omega_{c}\right]}\right\|_{2} .
$$

The above frequency components of $y$ were obtained via DFT and subsequent inverse DFT analysis for the corresponding frequency regions. To allow more transparent interpretation, (34) and (35) were scaled by $1 / \sqrt{N}$ to yield RMSE values, where $N$ is the total sample number $(=40 / T)$.

\section{RESUlTS}

The mean fitting accuracy of the model validation tests of Section V-B2 was 58.6\% across Phase 1 participants (Participants 1, 2, 3, 4), and 61.2\% for the PwMS in Phase 2. These were lower than the results of the previous study [20]. The extensive tests in [20] show that selection of higher order components within the model can be used to increase accuracy, however this comes at the price of needing much longer identification tests. Therefore it was decided to keep the aforementioned model forms in order to more thoroughly assess the robust performance of $\mathrm{RC}$ in suppressing tremor.

\section{A. Effectiveness of RC Algorithms on Tremor Suppression}

In this section, the first RC design (without employing the ZPHP filter $F_{H}$ ) is evaluated. A representative result from Phase 1 tracking Test $T 1$ is shown in Fig. 6 and confirms that voluntary action contains only frequencies contents below 1 $\mathrm{Hz}$, and results in accurate completion when tremor is absent. A representative result from tracking Test $T 2$ is also shown in Fig. 6 and confirms that the induced tremor manifests as an oscillation of $f_{p}=2 \mathrm{~Hz}$ in conjunction with voluntary action. Fig. 6 also shows wrist joint position data acquired from Tracking Test $T 3$. To quantify these results, values of the suppression and voluntary interference norm (34) and (35) respectively are given in Table I. For each participant, Tracking Test $T 3$ was repeated several times for different controller parameters to explore the balance between tremor suppression and voluntary interference. Tests were halted if the participant experienced any discomfort. To aid comparison, the results in Table I are sorted in descending order of $\Delta y_{t}$ without the ZPHP filter. Representative results for the PwMS in Phase 2 are shown in Fig. 7. Here Test $T 2$ shows tremulous motion manifests with frequency $f_{p}=2.4 \mathrm{~Hz}$.

The value of $\Delta y_{t}$ from Tracking Test $T 2$ indicates large tremulous movements (0.1407 in Participant $1,0.1790$ in Participant 2, 0.1249 in Participant 3, 0.2246 in Participant 4 and 0.1208 in PwMS). Phase 1 results show that FMI-RC and GB-RC applications result in an average suppression of $89.0 \%$ and $82.7 \%$ respectively, while the average tremor suppression provided by the HPF (designed in Section V-B2c) is $59.8 \%$ 


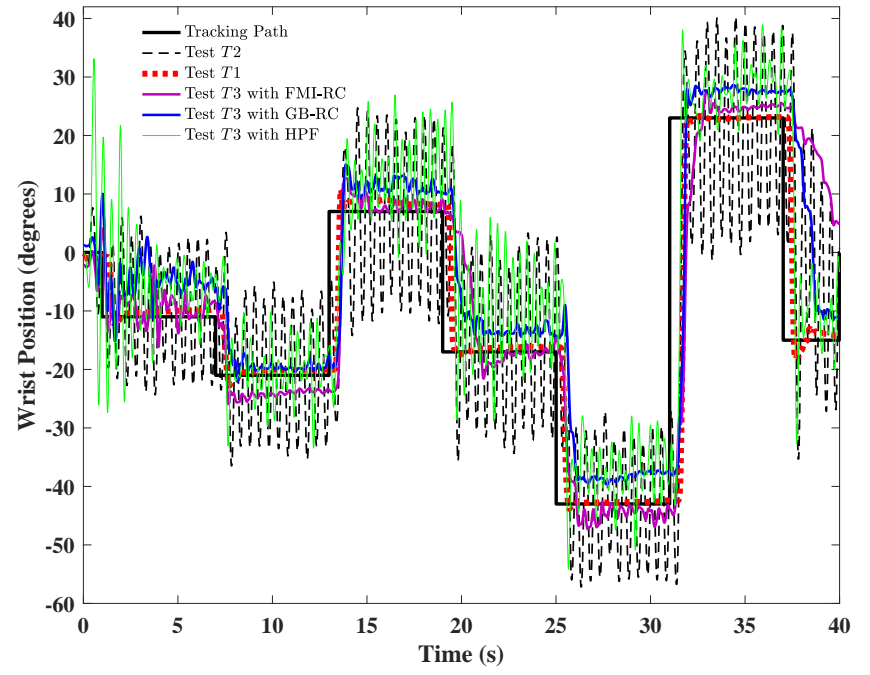

Fig. 6. Wrist angular positions from all tracking tests of Participant 1.

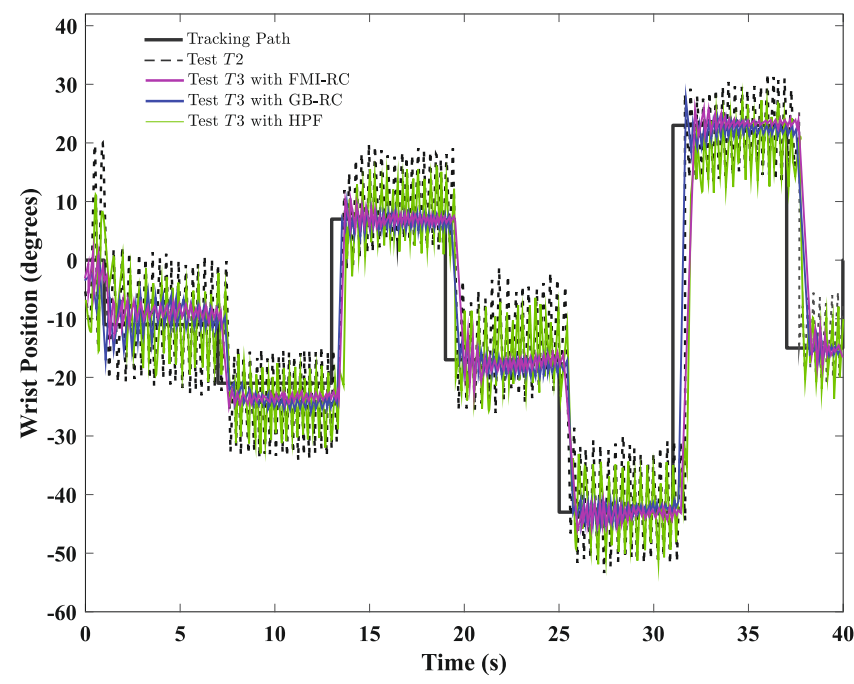

Fig. 7. Wrist angular positions from all tracking tests of PwMS.

confirming the advantage of RC. In Phase 2 tests with PwMS, FMI-RC and GB-RC suppress tremor by $88.4 \%$ and $80.7 \%$ respectively, and the HPF by $55 \%$.

In Participant 2, FMI-RC suppresses involuntary movements by up to $93 \%$ (reducing $\Delta y_{t}$ from 0.1790 to 0.0119 ) whilst GB-RC suppresses involuntary movements by up to $92 \%$ (reducing $\Delta y_{t}$ from 0.1790 to 0.0144 ). However, the largest suppression in tremor amplitude is less in other participants. In Participant 1, FMI-RC and GB-RC can suppress tremor by up to $91 \%$ (reducing $\Delta y_{t}$ from 0.1407 to 0.0128 ) and by up to $87 \%$ (reducing $\Delta y_{t}$ from 0.1407 to 0.0188 ), respectively. Tremor suppression provided by FMI-RC in Participant 4, which is found as $90 \%$ (reducing $\Delta y_{t}$ from 0.2266 to 0.0545 ), is very close to that in Participant 1 . The lowest suppression in FMI-RC approach is found as $89 \%$ in Participant 3 (reducing $\Delta y_{t}$ from 0.1249 to 0.0137$)$. In addition, GB-RC approach can suppress tremor in Participant 3 and Participant 4 by around $80 \%$. The PwMS results in Phase 2 show levels of suppression for FMI-RC of up to $89 \%$ (reducing $\Delta y_{t}$ from 0.1208 to
TABLE I

CHANGES IN THE SCALED MEASURES WITH VARIED CONTROL VARIABLES OF ALL CONTROLLERS.

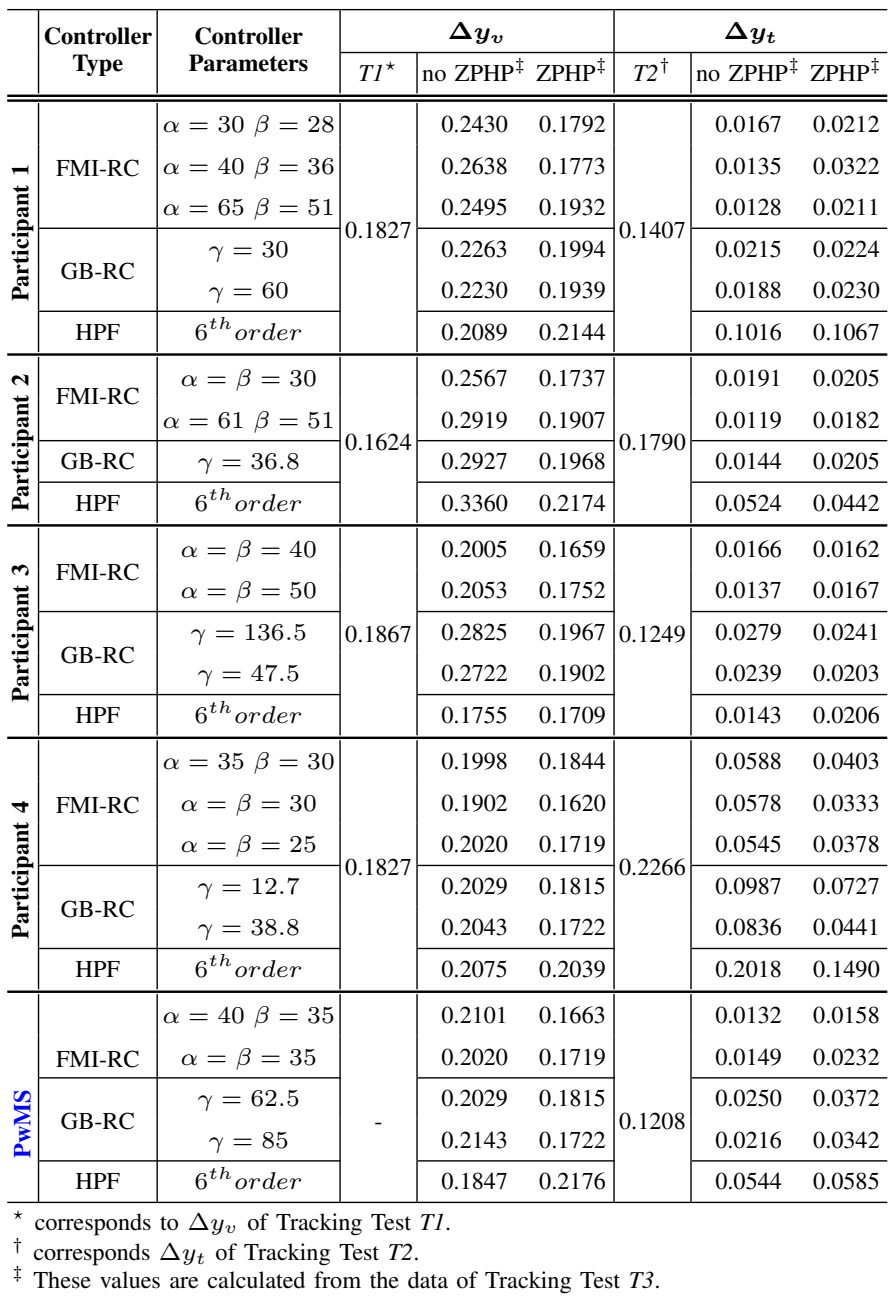

0.0132 ) and for GB-RC of up to $82 \%$ (reducing $\Delta y_{t}$ from 0.1208 to 0.0216 ). These are similar to the Phase 1 results.

Table I indicates that HPF exhibits a decrease of $71 \%$ (reducing $\Delta y_{t}$ from 0.1790 to 0.0524 ) and $51 \%$ (reducing $\Delta y_{t}$ from 0.2266 to 0.2018 ) in tremor amplitude in Participant 2 and Participant 4, respectively. In addition, the poorest performance of HPF is obtained in Participant 1 with the tremor suppression of $28 \%$ (reducing $\Delta y_{t}$ from 0.1407 to 0.1016 ) and the highest is obtained in Participant 3 with $89 \%$ (reducing $\Delta y_{t}$ from 0.1249 to 0.0143 ) tremor suppression. The PwMS result of $55 \%$ (reducing $\Delta y_{t}$ from 0.1208 to 0.0585 ) sits firmly within the range of Phase 1 results.

Table I also shows that $\Delta y_{v}$ corresponding to Tracking Test $T 1$ is 0.1827 in Participant 1, 0.1624 in Participant 2, 0.1867 in Participant 3 and 0.1827 in Participant 4. Comparison of these results with those relevant to the Tracking Test $T 3$ indicates that FMI-RC, GB-RC and HPF increase $\Delta y_{v}$ by an average of $29.3 \%, 35.2 \%$ and $33.7 \%$ in Phase 1 . This means that voluntary distortion is experienced in all control approaches, except HPF in Participant 3. In this one case $\Delta y_{v}$ is actually smaller than the baseline value from Tracking Test $T 1$, which may be due to participants learning how to track better over time. In Phase 2 FMI-RC, GB-RC and HPF increase $\Delta y_{v}$ by 
an average of $27 \%, 31 \%$ and $37 \%$ respectively.

In summary, these results confirm that RC can suppress tremor more than the leading conventional approach. However voluntary distortion is greater. The ability of the ZPHP filter to reduce this distortion will now be examined.

\section{B. Effects of ZPHP Filter on Voluntary Distortion}

In this section, the second RC design of Section V-B2 employing the ZPHP filter $F_{H}$ is evaluated to confirm properties of Theorem 4. From the data in Table I, it is apparent that adding the ZPHP filter contributes to a decrease in $\Delta y_{v}$ in all cases of RC: for Phase 1 the average decrease in $\Delta y_{v}$ are $21.8 \%$ and $18.7 \%$ for FMI-RC and GB-RC respectively. In contrast, there is only one significant reduction when the ZPHP filter is used with HPF, which is for Participant 2 (the same participant where the highest decrease in $\Delta y_{v}$ occurred for the $\mathrm{RC}$ approaches). Phase 2 results are similar: the average decrease in $\Delta y_{v}$ are $18 \%$ and $15 \%$ for FMI-RC and GB-RC respectively, and no improvement occurs for the HPF case.

The results show that $\Delta y_{v}$ is decreased by an average of $22.7 \%$ and $33.5 \%$ in Participant 1 and Participant 2 for FMIRC cases. In addition, ZPHP filter can produce a decrease of $32.8 \%$ and $30.4 \%$ in $\Delta y_{v}$ in Participant 2 and Participant 3 for GB-RC cases. In Participant 4 , the decrease in $\Delta y_{v}$ ranges from $7.7 \%$ to $15.7 \%$ for RC cases. Thus these results confirm that the ZPHP filter is able to reduce the interference of RC approaches with voluntary action. However the ZPHP filter may cause a slight increase in $\Delta y_{t}$ in some cases since adding the ZPHP filter shapes the frequency response of $S(q)$ and leads to a slight increase in the gain of $|S(q)|$ at the tremor frequency. In contrast, in several cases the ZPHP filter actually leads to a reduction in $\Delta y_{t}$ (see e.g. Participant 4). Fig. 8 contains representative results showing that $\mathrm{RC}$ can suppress tremor effectively when the ZPHP filter is added. These results illustrate that adding a filter enables faster and more accurate tracking due to the lack of interference.

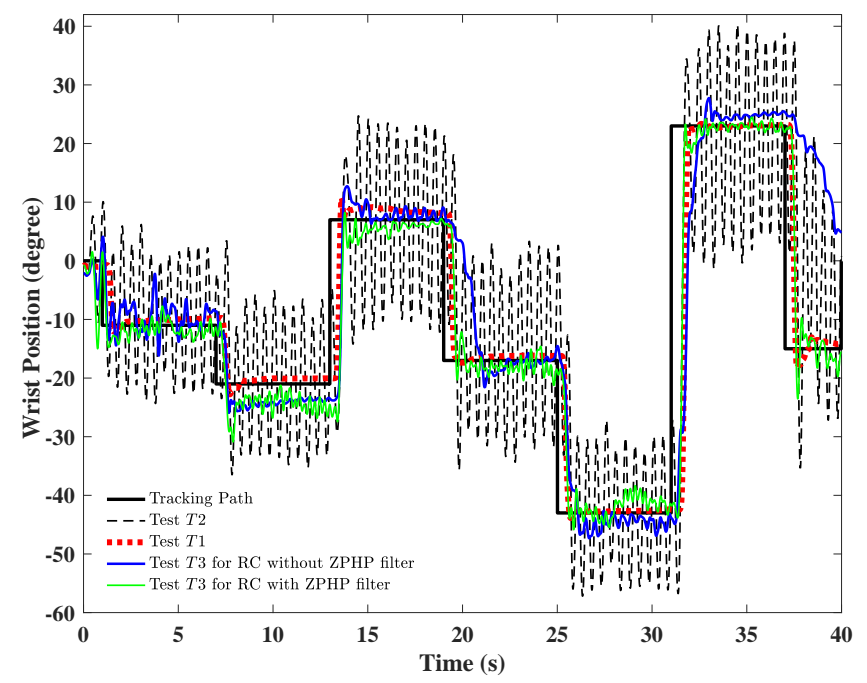

Fig. 8. Wrist angular positions of the closed-loop RC system without and with ZPHP filter from Participant 1.

\section{CONCLUSION}

The aim of this study was first to develop a feedback RC system for suppressing an induced tremor at a single peak frequency by means of FES. The second aim was to establish its effect on patients' voluntary motion, and subsequently eliminate this interference. A framework has been developed which enables a wide range of $\mathrm{RC}$ schemes to be employed for tremor suppression. This framework comprises a model structure, linearising controller, identification procedure and a mechanism to preserve voluntary action. A clinically relevant system identification procedure has firstly been developed including both non-linear and linear dynamics. Then two linear RC algorithms, GB-RC and FMI-RC, have been designed based on the identified model and tested using a wrist rig. An experimental study evaluated the approach with both neurologically intact and neurologically impaired participants. This assessed the ability of RC algorithms for suppressing tremor and their effect on voluntary motion.

The test results show that RC is more effective than conventional filtering techniques for tremor suppression. However $\mathrm{RC}$ was shown to interfere with voluntary motion, leading to impairment in wrist movements. Thus a ZPHP filter was designed and implemented to reduce this distortion in the intended movement. The experiments confirmed that the inclusion of the ZPHP filter led to minimal interference in voluntary motion but may cause a mild reduction in tremor suppression. Compared with results in [46] using the previous RC approach whose aim was purely to show feasibility, the results confirm significant improvement in tremor suppression (from $12 \%$ to over $80 \%$ ), as well as reduced distortion of voluntary motion. This has been achieved by applying the comprehensive design and implementation framework developed in this paper.

Future work will focus on extending the model to include the elbow and shoulder joints, using forms that have previously proved successfully in FES assistive technology, e.g. [47]. The proposed control approach can be applied to this case by expanding the linearizing control component to embed state feedback or input-output linearisation action, prior to MIMO RC design. In this way, the same philosophy naturally extends to the entire upper limb (e.g. both FMI-RC and GB-RC transparently extend to MIMO dynamics). Such expansions to more complex, coupled dynamics inevitably amplify the effect of model uncertainty. This can be addressed by selecting and tuning the RC approaches to focus on robustness rather than convergence speed, as has been discussed in this paper.

A related issue is to embed robustness to an unknown or varying tremor frequency. This may be tackled by adding additional RC update terms of the form considered in this paper, each designed for a slightly different tremor frequency [48]. Analysis then shows how how the range of stabilized frequencies correspondingly enlarges. Alternatively, the estimation-based multiple-model switched adaptive RC (MMRC) approach of [49] can be applied. This can guarantee stability for an arbitrary amount of uncertainty in the underlying dynamics and also in the tremor frequency. The MMRC structure encompasses a bank of RC controllers (each designed for a different model, following the procedure in this 
paper), and an associated bank of Kalman filters which decide on which controller is switched into closed loop. MMRC then automatically switches in the controller that corresponds to the model and tremor frequency that best fit the observed data (i.e. with no need for further identification or tuning).

More extensive clinical testing will be undertaken with MS patients diagnosed with intention tremor in order to verify the effectiveness of the proposed approach with end-users. Then future work will focus on developing a wearable tremor suppression system. This will exploit recent developments in fabric FES electrodes [50], miniature electronics, and sensors.

\section{REFERENCES}

[1] M. Koch, J. Mostert, D. Heersema, and J. De Keyser, "Tremor in multiple sclerosis," Journal of Neurology, vol. 254, no. 2, pp. 133-145, 2007.

[2] G. Deuschl, P. Bain, M. Brin, and an Ad Hoc Scientific Committee, "Consensus statement of the movement disorder society on tremor," Movement Disorders, vol. 13, no. 3, pp. 2-23, 1998.

[3] G. Deuschl, J. Raethjen, M. Lindemann, and P. Krack, "The pathophysiology of tremor," Muscle \& Nerve, vol. 24, no. 6, pp. 716-735, 2001. [Online]. Available: http://dx.doi.org/10.1002/mus.1063

[4] J. McGruder, D. Cors, A. M. Tiernan, and G. Tomlin, "Weighted wrist cuffs for tremor reduction during eating in adults with static brain lesions," American Journal of Occupational Therapy, vol. 57, no. 5, pp. 507-516, 2003.

[5] A. Hassan, J. E. Ahlskog, M. Rodriguez, and J. Y. Matsumoto, "Surgical therapy for multiple sclerosis tremor: a 12-year follow-up study," European Journal of Neurology, vol. 19, no. 5, pp. 764-768, 2012.

[6] P. R. Schuurman, D. A. Bosch, P. M. M. Bossuyt, G. J. Bonsel, E. J. W. Van Someren, R. M. A. De Bie, M. P. Merkus, and J. D. Speelman, "A comparison of continuos thalamic stimulation and thalamotomy for suppression of severe tremor," The New England Journal of Medicine, vol. 342, no. 7, pp. 461-468, 2000.

[7] L. Yap, A. Kouyialis, and T. R. K. Varma, "Stereotactic neurosurgery for disabling tremor in multiple sclerosis: thalamotomy or deep brain stimulation?," British Journal of Neurosurgery, vol. 21, no. 4, pp. 349 354, 2007.

[8] M. Heenan, R. A. Scheidt, D. Woo, and S. A. Beardsley, "Intention tremor and deficits of sensory feedback control in multiple sclerosis: a pilot study," Journal of NeuroEngineering and Rehabilitation, vol. 11, no. 1, pp. 1-19, 2014.

[9] E. Rocon, J. A. Gallego, B.-L. J. M., J. Benito-Leon, and L. P. J., "Biomechanical loading as an alternative treatment for tremor: a review of two approaches," Tremor and Other Hyperkinetic Movements, vol. 2, pp. 1-13, 2012.

[10] S. Pledgie and K. E. Barner, "Tremor suppression through impedance control," IEEE Transactions on Rehabilitation Engineering, vol. 8, no. 1, pp. 53-59, 2000.

[11] P. Feys, W. Helsen, X. Liu, D. Mooren, H. Albrecht, B. Nuttin, and P. Ketelaer, "Effects of peripheral cooling on intention tremor in multiple sclerosis," Journal of Neurology, Neurosurgery and Psychiatry, vol. 76, no. 3, pp. 373-379, 2005.

[12] P. Feys, W. F. Helsen, S. Verschueren, S. P. Swinnen, I. Klok, A. Lavrysen, B. Nuttin, P. Ketelaer, and X. Liu, "Online movement control in multiple sclerosis patients with tremor: effects of tendon vibration," Movement Disorders, vol. 21, no. 8, pp. 1148-1153, 2006.

[13] F. Hawes, C. Billups, and S. Forwell, "Interventions for upper-limb intention tremor in multiple sclerosis," International Journal of MS Care, vol. 12, no. 3, pp. 122-132, 2010.

[14] A. Prochazka, J. Elek, and M. Javidan, "Attenuation of pathological tremors by functional electrical stimulation. II: Clinical evaluation," Annals of Biomedical Engineering, vol. 20, no. 2, pp. 225-236, 1992.

[15] D. Zhang and W. T. Ang, "Tremor suppression of elbow joint via functional electrical stimulation: a simulation study," in Proceeding of the 2006 IEEE International Conference on Automation Science and Engineering, 2006, pp. 182-187.

[16] A. P. L. Bo, C. Azevedo-Coste, P. Poignet, C. Geny, and C. Fattal, "On the use of FES to attenuate tremor by modulating joint impedance," in 50th IEEE Conference on Decision and Control and European Control Conference, 2011, pp. 6498-6503.
[17] J. Á. Gallego, E. Rocon, J. M. Belda-Lois, and J. L. Pons, "A neuroprosthesis for tremor management through the control of muscle cocontraction," Journal of NeuroEngineering and Rehabilitation, vol. 10, no. 1, pp. 1-13, 2013.

[18] A. Prochazka, J. Elek, and M. Javidan, "Attenuation of pathological tremors by functional electrical stimulation. I: Method," Annals of Biomedical Engineering, vol. 20, no. 2, pp. 205-224, 1992.

[19] B. A. Francis and W. M. Wonham, "The internal model principle for linear multivariable regulators," Applied Mathematics and Optimization, vol. 2, no. 2, pp. 170-194, 1975.

[20] E. H. Copur, C. T. Freeman, B. Chu, and D. S. Laila, "System identification for FES-based tremor suppression," European Journal of Control, vol. 27, pp. 45-59, 2016.

[21] C. T. Freeman, P. A. Sampson, J. H. Burridge, and A. M. Hughes, "Repetitive control of functional electrical stimulation for induced tremor suppression," Mechatronics, vol. 32, pp. 79-87, 2015.

[22] P. G. Bain, "The management of tremor," Journal of Neurology, Neurosurgery \& Psychiatry, vol. 72, no. suppl 1, pp. i3-i9, 2002.

[23] G. Grimaldi and M.-U. Manto, Tremor: From Pathogenesis To Treatment. San Rafael, California: Morgan \& Claypool, 2008.

[24] E.-W. Bai, Z. Cai, S. Dudley-Javoroskv, and R. K. Shields, "Identification of a modified Wiener-Hammerstein system and its application in electrically stimulated paralyzed skeletal muscle modeling," Automatica, vol. 45, pp. 736-743, 2009.

[25] J. Bobet, E. Gossen, and R. Stein, "A comparison of models of force production during stimulated isometric ankle dorsiflexion in humans," IEEE Transactions on Neural Systems and Rehabilitation Engineering, vol. 13, no. 4, pp. 444-451, 2005.

[26] W. Durfee and K. Palmer, "Estimation of force-activation, force-length, and force-velocity properties in isolated, electrically stimulated muscle,' IEEE Transactions on Biomedical Engineering, vol. 41, no. 3, pp. 205216, 1994.

[27] F. Le, I. Markovsky, C. T. Freeman, and E. Rogers, "Identification of electrically stimulated muscle models of stroke patients," Control Engineering Practice, vol. 18, no. 4, pp. 396-407, 2010.

[28] M. Curtin and M. M. Lowery, "Musculoskeletal modelling of muscle activation and applied external forces for the correction of scoliosis," Journal of Neuroengineering and Rehabilitation, vol. 11, no. 1, p. 52, 2014.

[29] J. M. Iaquinto and J. S. Wayne, "Computational model of the lower leg and foot/ankle complex: application to arch stability," Journal of Biomechanical Engineering, vol. 132, no. 2, p. 021009, 2010.

[30] S. Takehara, M. Murakami, and K. Hase, "Biomechanical evaluation of an electric power-assisted bicycle by a musculoskeletal model," Journal of System Design and Dynamics, vol. 6, no. 3, pp. 343-350, 2012.

[31] G. M. Lyons, G. E. Leane, M. Clarke-Moloney, J. V. O’Brien, and P. A. Grace, "An investigation of the effect of electrode size and electrode location on comfort during stimulation of the gastrocnemius muscle," Medical Engineering and Physics, vol. 26, no. 10, pp. 873 - 878, 2004.

[32] N. Hogan, "Adaptive control of mechanical impedance by coactivation of antagonist muscles," IEEE Transactions on Automatic Control, vol. 29, no. 8, pp. 681-690, 1984.

[33] R. W. Simmons and C. Richardson, "Peripheral regulation of stiffness during arm movements by coactivation of the antagonist muscles," Brain Research, vol. 473, no. 1, pp. 134-140, 1988.

[34] E. Langzam, Y. Nemirovsky, E. Isakov, and J. Mizrahi, "Partition between volitional and induced forces in electrically augmented dynamic isometric muscle contractions," IEEE Transactions on Neual Systems and Rehabilitation Engineering, vol. 13, no. 3, 2006.

[35] J. J. Hatonen, C. T. Freeman, D. H. Owens, P. L. Lewin, and E. Rogers, "A gradient-based repetitive control algorithm combining ILC and pole placement," European Journal of Control, vol. 12, no. 3, pp. 278-292, 2006.

[36] R. W. Longman, "Iterative learning control and repetitive control for engineering practice," International Journal of Control, vol. 73, no. 10, pp. 930-954, 2000.

[37] - "On the theory and design of linear repetitve control systems," European Journal of Control, vol. 16, no. 5, pp. 447-496, 2010.

[38] B. Panomruttanarug and R. W. Longman, "Repetitive controller design using optimization in the frequency domain," in Proceedings of the 2004 AIAA/AAS Astrodynamics Specialist Conference, 2004, pp. 1215-1236.

[39] J. J. Hatonen, C. T. Freeman, D. H. Owens, P. L. Lewin, and E. Rogers, "A gradient-based repetitive control algorithm combining ILC and pole placement," European Journal of Control, vol. 12, no. 3, pp. 278-292, 2006. 
[40] R. N. Stiles and D. W. Hahs, "Muscle-Load Oscillations: Detection, Analysis, and Models," in Bioinstrumentation and Biosensors. CRC Press, 1991, pp. 75-120.

[41] Y. Han, D. Owens, and B. Chu, "Linear optimal multi-periodic repetitive control - a low order controller scheme," An International Journal of Optimization and Control: Theories and Applications, vol. 2, no. 1, 2011.

[42] R. Turk, S. V. Notley, R. M. Pickering, D. M. Simpson, P. A. Wright, and J. H. Burridge, "Reliability and sensitivity of a wrist rig to measure motor control and spasticity in post-stroke hemiplegia," Neurorehabilitation and Neural Repair, vol. 22, no. 6, pp. 684-696, 2008.

[43] B. Freriks, H. J. Hermens, C. Disselhorst-Klug, and G. Rau, SENIAM 8: European Recommendations for Surface ElectroMyoGraphy. Roessingh Research and Development, 1999, ch. 2: The Recommendations for Sensors and Sensor Placement Procedures for Surface ElectroMyoGraphy.

[44] F. A. Lenz, C. J. Jaeger, M. S. Seike, Y. C. Lin, and S. G. Reich, "Singleneuron analysis of human thalamus in patients with intention tremor and other clinical signs of cerebellar disease," Journal of Neurophysiology, vol. 87, no. 4, pp. 2084-2094, 2002.

[45] C. L. Lynch and M. R. Popovic, "Functional electrical stimulation," IEEE Control System Magazine, vol. 28, no. 2, pp. 40-50, 2008.

[46] C. T. Freeman, P. A. Sampson, J. H. Burridge, and A.-M. Hughes, "Repetitive control of functional electrical stimulation for induced tremor suppression," Mechatronics, vol. 32, pp. 79-87, 2015.

[47] C. T. Freeman, "Upper limb electrical stimulation using input-output linearization and iterative learning control," IEEE Transactions on Control Systems Technology, vol. 23, no. 4, pp. 1546-1554, 2015.

[48] M. Steinbuch, "Repetitive control for systems with uncertain periodtime," Automatica, vol. 38, no. 12, pp. 2103-2109, 2002.

[49] C. T. Freeman, "Multiple model switched repetitive control," in Proceeding of the 2017 IEEE Conference on Decision and Control, 2017.

[50] K. Yang, C. T. Freeman, R. N. Torah, S. P. Beeby, and J. Tudor, "Screen printed fabric electrode array for wearable functional electrical stimulation," Sensors and Actuators A: Physical, vol. 213, pp. 108-115, 2014. 